\title{
The Role of Functional Dopamine-Transporter SPECT Imaging in Parkinsonian Syndromes, Part 2
}

\author{
T.C. Booth, M. Nathan, A.D. Waldman, A.-M. Quigley, A.H. Schapira, and J. Buscombe
}

\begin{abstract}
SUMMARY: The functional imaging technique most widely used in European clinics to differentiate a true parkinsonian syndrome from vascular parkinsonism, drug-induced changes, or essential tremor is dopamine-transporter SPECT. This technique commonly reports dopamine-transporter function, with decreasing striatal uptake demonstrating increasingly severe disease. The strength of dopaminetransporter SPECT is that nigrostriatal degeneration is observed in both clinically inconclusive parkinsonism and early, even premotor, disease. In this clinical review (Part 2), we present the dopamine-transporter SPECT findings in a variety of neurodegenerative diseases, including multiple system atrophy, progressive supranuclear palsy, corticobasal degeneration, and dementia with Lewy bodies. The findings in vascular parkinsonism, drug-induced parkinsonism, and essential tremor are also described. It is hoped that this technique will be the forerunner of a range of routinely used, process-specific ligands that can identify early degenerative disease and subsequently guide disease-modifying interventions.
\end{abstract}

ABBREVIATIONS: $\mathrm{CBD}=$ corticobasal degeneration; $\mathrm{DaT}=$ dopamine transporters; ${ }^{18} \mathrm{~F}=$ fluorine $18 ; \mathrm{IBF}={ }^{123} \mathrm{I}-(\mathrm{S})-5$-iodo-7- $\mathrm{N}-\{(1-\mathrm{ethyl}-2$-pyrrolidinyl) methyl\}carboxamido-2,3-dihydrobenzofuran; IBZM = ${ }^{123}$ I-S-(K)-N-[(1-ethyl-2-pyrrolidinyl)methyl]-2-hydroxy-3-iodo-6-methoxybenzamide; ${ }^{123}$ - $-\mathrm{FP}$-CIT = ${ }^{123}$-ioflupane $(\mathrm{N}$ - $\omega$-fluoropropyl- $\beta \mathrm{CIT}) ; \mathrm{MSA}=$ multiple system atrophy; MSA-P = patients with multiple system atrophy with predominant parkinsonism; PSP $=$ progressive supranuclear palsy

$T^{1}$ he dopamine transporter is a sodium chloride-dependent transmembrane protein localized to the presynaptic nigrostriatal cell surface. SPECT imaging of dopamine transporters (DaT) with specific tracers gives a guide to the attenuation of functional dopaminergic neurons. The technical concepts of DaT-SPECT were outlined in Part 1 (The Role of Functional Dopamine Transporter SPECT Imaging in Parkinsonian Syndromes, Part 1) of this 2-part Review Article and would be valuable reading as background to this article.

What appears to be the strength of DaT-SPECT, like 6 - $\left[{ }^{18} \mathrm{~F}\right]$-fluoro-L-3,4-dihydroxyphenylalanine PET, is that nigrostriatal degeneration is observed in both clinically inconclusive parkinsonism and early, even premotor, disease. DaTSPECT images are easy to interpret compared with advanced

From the Department of Neuroradiology (T.C.B.), National Hospital for Neurology and Neurosurgery, London, UK; Department of Nuclear Medicine (M.N., A.-M.Q.), Royal Free Hospital NHS Trust, London, UK; Department of Imaging (A.D.W.), Imperial College Healthcare NHS Trust, London, UK; Department of Clinical Neurosciences (A.H.S.), Institute of Neurology, University College London, London, UK; and Department of Nuclear Medicine (J.B.), Addenbrooke's Hospital, Cambridge University Hospitals NHS Foundation Trust, Cambridge, UK.

Please address correspondence to Thomas C. Booth, MA, MBBS, MRCP, FRCR, Department of Neuroradiology, National Hospital for Neurology and Neurosurgery, Queen Square, London, WCIN 3BG, UK; e-mail: tombooth@doctors.org.uk

- Indicates open access to non-subscribers at www.ajnr.org

http://dx.doi.org/10.3174/ajnr.A3971
MR imaging techniques; the cost is low compared with that of PET; all compliant patients can be imaged, unlike in transcranial sonography; and at least in Europe, SPECT is widely available compared with PET, advanced MR imaging, and transcranial sonography.

Parkinsonian syndromes are a group of movement disorders characterized by tremor, bradykinesia, and rigidity. They are most frequently due to primary neurodegenerative disease, resulting in loss of dopaminergic nerve terminals along the nigrostriatal pathway, as occurs in idiopathic Parkinson disease, multiple system atrophy (MSA), progressive supranuclear palsy (PSP), corticobasal degeneration (CBD), and dementia with Lewy bodies. Other causes of parkinsonism that do not involve nigrostriatal degeneration include drug-induced, vascular or toxic insult, and psychogenic disease. Patients with nigrostriatal degeneration may benefit from dopaminergic medication, whereas nondegenerative or postsynaptic etiologies do not.

The Table demonstrates the distinguishing clinical features of idiopathic Parkinson disease, MSA, PSP, CBD, and dementia with Lewy bodies. Due to its multisystem involvement, patients with MSA are typically subdivided into those who present with predominant parkinsonism (MSA-P) and those with predominant cerebellar ataxia (MSA-C). The clinical diagnosis of idiopathic Parkinson disease, based on clinical signs, assessment of inclusion and exclusion criteria, and good response to 


\begin{tabular}{|c|c|c|}
\hline & $\begin{array}{l}\text { Clinical Features of Parkinsonism } \\
\text { (Bradykinesia, Rigidity, Tremor) }\end{array}$ & Additional Helpful Clinical Features \\
\hline \multicolumn{3}{|l|}{ Neurodegenerative Parkinsonism } \\
\hline Parkinson disease & Yes & Nil \\
\hline Multiple system atrophy & Yes & Pyramidal, autonomic, and cerebellar signs \\
\hline Progressive supranuclear palsy & Yes & $\begin{array}{l}\text { Supranuclear gaze palsy, increased axial tone, } \\
\text { bulbar palsy, early postural instability }\end{array}$ \\
\hline Corticobasal degeneration & Yes & Asymmetric cortical dysfunction \\
\hline Dementia with Lewy bodies & Yes & $\begin{array}{l}\text { Fluctuating dementia, visual hallucinations, } \\
\text { hypersensitivity to neuroleptic medications }\end{array}$ \\
\hline \multicolumn{3}{|c|}{$\begin{array}{l}\text { Non-neurodegenerative etiologies of } \\
\text { parkinsonism }\end{array}$} \\
\hline Essential tremor & Tremor & $\begin{array}{l}\text { Usually bilateral tremor that worsens on } \\
\text { movement; tremor can affect head and voice }\end{array}$ \\
\hline Vascular parkinsonism & $\begin{array}{l}\text { Bradykinesia with at least one of resting } \\
\text { tremor, rigidity, or postural instability }\end{array}$ & $\begin{array}{l}\text { Cerebrovascular disease diagnosed clinically or on } \\
\text { CT/MR imaging; temporal relationship between } \\
\text { cerebrovascular infarcts near or within the basal } \\
\text { ganglia and acute/progressive development of } \\
\text { parkinsonism; insidious onset of parkinsonism } \\
\text { with bilateral symptoms at onset, presence of } \\
\text { early shuffling gait, or cognitive dysfunction and } \\
\text { extensive subcortical white matter lesions on MRI }\end{array}$ \\
\hline Drug-induced parkinsonism & Yes & $\begin{array}{l}\text { Very difficult to distinguish clinically from } \\
\text { neurodegenerative idiopathic Parkinson disease }\end{array}$ \\
\hline
\end{tabular}

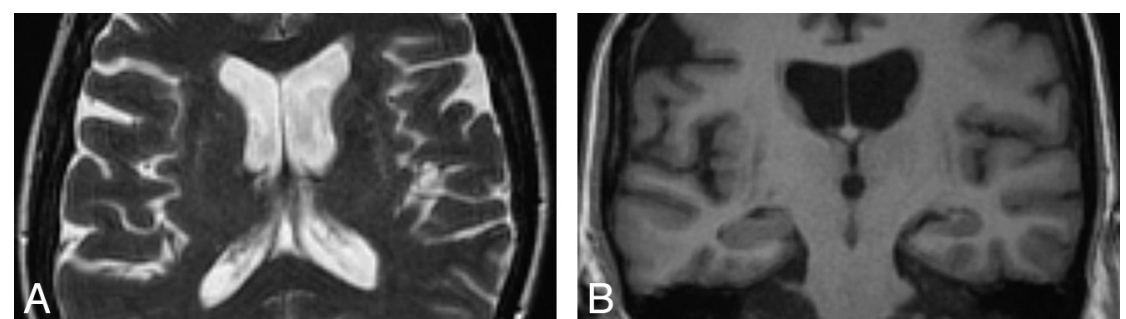

FIG 1. Axial T2-weighted MR imaging sequence at $1.5 T$ in a patient with MSA-P $(A)$ demonstrating hyperintense putaminal rims. The sign is $96 \%$ specific in differentiating patients with MSA from those with idiopathic Parkinson disease; however, it is only $56 \%$ sensitive according to 1 study. ${ }^{6}$ The corresponding coronal T1-weighted MR imaging sequence $(B)$ shows that the putaminal rims are hypointense. The putaminal rim sign is nonspecific in the wider population-for example, it can be seen in Wilson disease or some spinocerebellar ataxia subtypes. It is also a normal finding at 3T.

levodopa, ${ }^{1}$ can be straightforward. However, mild, atypical, or ambiguous signs with unconvincing responses to levodopa, can make the diagnosis inconclusive. ${ }^{2}$ This may have adverse prognostic implications in the management of parkinsonism.

Another important clinical differential diagnosis is essential tremor. Typical cases of essential tremor are easily distinguished from idiopathic Parkinson disease, but diagnosis may be more of a challenge if the tremor is unilateral or if there is a dystonic upper limb tremor. Where there is clinical doubt, functional DaTSPECT imaging may be decisive.

\section{Multiple System Atrophy}

Approximately $10 \%$ of patients with parkinsonism are diagnosed with MSA, ${ }^{3}$ which is characterized by widespread neurodegeneration, especially within the striatum, substantia nigra, pons, inferior olivary and vagal motor nuclei, cerebellum, and spinal cord. ${ }^{4}$

Structural MR imaging findings overlap in all MSA subtypes, independent of clinical presentation, though trends can be seen. In MSA-P, the putamen may demonstrate a rim of increased sig- nal on T2-weighted sequences due to gliosis (Fig 1). ${ }^{5}$ In MSA with predominant cerebellar ataxia, pontocerebellar degeneration may result in visually apparent cerebellar and pontine atrophy, which, along with MSA-P to a lesser extent, is associated with increased signal on T2-weighted sequences in the middle cerebellar peduncles, cerebellum, and the pontine transverse fibers and raphe ("hot cross bun" sign) (Fig 2).

Atrophy is most evident in patients with well-established disease. If one uses a cutoff value of $8 \mathrm{~mm}$, the middle cerebellar peduncle width allows differentiation of patients with MSA compared with controls or those with idiopathic Parkinson disease with $100 \%$ positive predictive value (Fig 2). ${ }^{6}$ Volumetry and voxel-based morphometry group studies show striatal and cerebellar volume loss in patients with MSA-P compared with those with idiopathic Parkinson disease and controls. ${ }^{7-9}$ Voxel-based morphometry also demonstrates selective cortical atrophy in MSA-P, affecting the motor cortical targets of basal ganglia output pathways such as the primary sensorimotor and lateral premotor cortices and the prefrontal and insular cortices. $^{8}$

Although validation in prospective studies is required, high regional ADC in patients with MSA-P appears discriminatory. With $100 \%$ positive predictive value, putaminal ADC differentiates MSA-P and idiopathic Parkinson disease, ${ }^{10}$ whereas middle cerebellar peduncle ADC distinguishes MSA-P from PSP and idiopathic Parkinson disease. ${ }^{11}$ Similarly, when combined with DTI, high ADC and low fractional anisotropy in either the pons, cerebellum, or putamen are demonstrated in all patients with MSA-P, allowing this disease to be distinguished from idiopathic Parkinson disease. ${ }^{12}$ 


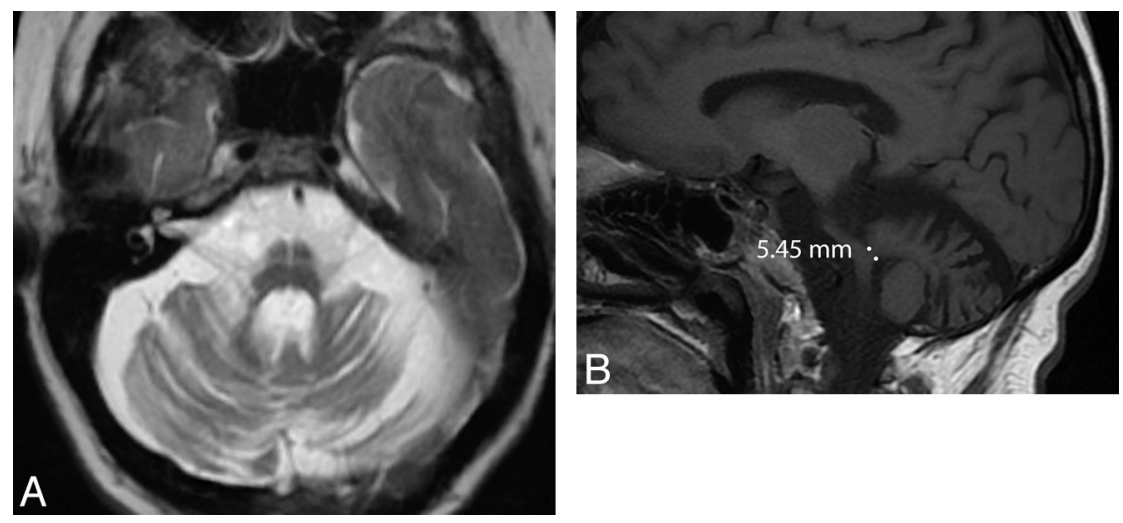

FIG 2. Axial T2-weighted MR imaging sequence in a patient with MSA with predominant cerebellar ataxia $(A)$ demonstrating the "hot-cross bun" sign, which results from selective loss of myelinated pontine transverse fibers and raphe neurons. One study showed that the sign is $100 \%$ specific in differentiating patients with MSA from those with idiopathic Parkinson disease; however, it is only $50 \%$ sensitive. ${ }^{6}$ Marked pontine and cerebellar atrophy is also demonstrated. This olivopontocerebellar volume loss is shown on the sagittal Tl-weighted sequences (B), where a middle cerebellar peduncle width $<8 \mathrm{~mm}$ is demonstrated. All these signs are nonspecific in the wider population-for example, they can be seen in some spinocerebellar ataxia subtypes.

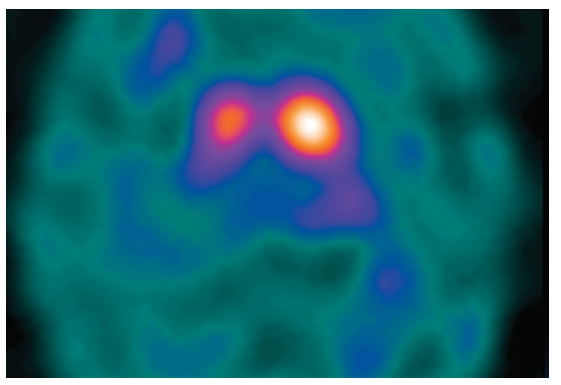

FIG 3. Axial ${ }^{123} \mathrm{I}-\mathrm{FP}-\mathrm{CIT}$ DaT-SPECT image in a patient with MSA. Note that imaging appearances are similar to those in idiopathic Parkinson disease, with asymmetric reduction in putaminal uptake. It is, therefore, difficult to differentiate MSA and idiopathic Parkinson disease on the basis of DaT-SPECT imaging alone.

Although less impressive than diffusion-based strategies, at $3 \mathrm{~T}^{1} \mathrm{H}-$ MRS, combined assessment of the NAA/Cr ratio in the pontine base and putamen may be effective in differentiating MSA-P from idiopathic Parkinson disease. ${ }^{13}$

With transcranial sonography, a combination of lenticular hyperechogenicity and normal echogenicity of the substantia nigra distinguishes MSA-P from idiopathic Parkinson disease with a positive predictive value of $100 \% .{ }^{14}$ With $6-\left[{ }^{18} \mathrm{~F}\right]$ fluoroL-3,4-dihydroxyphenylalanine PET, MSA may be indistinguishable from idiopathic Parkinson disease or may show lower caudate uptake. ${ }^{15,16}$ PET studies also show reduced striatal dopamine-receptor binding and ${ }^{18} \mathrm{~F}$-FDG uptake in MSA, with 1 study suggesting that MSA can be distinguished from idiopathic Parkinson disease with $100 \%$ positive predictive value. ${ }^{7,16}$

\section{DaT-SPECT in Multiple System Atrophy}

There is no discernible difference in the pattern of nigrostriatal degeneration between idiopathic Parkinson disease and MSA. This is reflected in DaT-SPECT imaging in which abnormal scan findings demonstrating asymmetric reduction in DaT binding are consistent with either disease (Fig 3). ${ }^{17,18}$ Recently, DaT-SPECT voxel-based morphometry has been used at the group level to distinguish MSA-P from idiopathic Parkinson disease. ${ }^{19}$ In the dorsal brain stem, there was greater signal reduction of patients with MSA-P compared with those with idiopathic Parkinson disease, and after a mean follow-up of 2.4 years, there was a greater signal reduction in the caudate and anterior putamen in patients with MSA-P compared with those with idiopathic Parkinson disease. This is in accordance with faster disease progression.

Similar to those who investigated PET, researchers have endeavored to distinguish idiopathic Parkinson disease and MSA by using SPECT dopamine $\mathrm{D}_{2}$ receptor imaging. Most striatal $\mathrm{D}_{2}$ receptors are located postsynaptically and function by facilitating cell-to-cell communication. They have a key role in modulating locomotion and are a critical target for dopaminergic therapeutics. ${ }^{20}$ SPECT radiolabeled ligands used to image $\mathrm{D}_{2}$ receptors are dopaminereceptor antagonists such as ${ }^{123} \mathrm{I}-\mathrm{S}-(\mathrm{K})-\mathrm{N}-[(1-$ ethyl2-pyrrolidinyl)methyl]-2-hydroxy-3-iodo-6-methoxybenzamide $(\text { IBZM })^{21}$ and ${ }^{123} \mathrm{I}-(S)-5$-iodo-7- $N$-\{(1-ethyl-2-pyrrolidinyl) methyl\}carboxamido-2,3-dihydrobenzofuran (IBF). ${ }^{22}$ A neuropathologic study has demonstrated loss of striatal $\mathrm{D}_{2}$ receptors in patients with $\mathrm{MSA}^{23}$ and there is corroborative evidence on SPECT $^{24}$ and PET as described above. Therefore, in contrast to patients with idiopathic Parkinson disease who do not have reduced $\mathrm{D}_{2}$ receptor binding, patients with MSA may demonstrate a reduction in $\mathrm{D}_{2}$ receptors. ${ }^{25}$ This feature may also explain why patients with MSA do not show a noteworthy response to dopaminergic medication that binds to $\mathrm{D}_{2}$ receptors. Several studies have sought to differentiate idiopathic Parkinson disease from MSA or PSP by combining presynaptic DaT-SPECT with postsynaptic $\mathrm{D}_{2}$ imaging by using IBZM or IBF, and these are summarized below.

\section{Progressive Supranuclear Palsy}

PSP accounts for a small minority of patients with parkinsonism. Neuropathologically, this tauopathy is characterized by neuronal degeneration of the nigrostriatal pathway, basal ganglia, and brain stem nuclei with deposition of neurofibrillary tangles. A number of MR imaging features suggestive of PSP have been described, such as dilation of the third ventricle and signal increase of the midbrain. ${ }^{5,26}$ Although often absent, atrophy of the red nucleus, frontal and temporal lobe, and an anteroposterior midbrain diameter of $<17 \mathrm{~mm}$ can each differentiate PSP from MSA with at least $75 \%$ positive predictive value (Fig 4). If one uses a "MR parkinsonism index" [ (pons/ midbrain) • (middle cerebellar peduncle/superior cerebellar peduncle)], PSP can be prospectively distinguished from idiopathic Parkinson disease, MSA-P, and controls with 100\% positive predictive value. ${ }^{27}$ Group studies additionally demonstrate that atrophy in the striatum and frontotemporal cortices, measured with volumetry and voxel-based mor- 

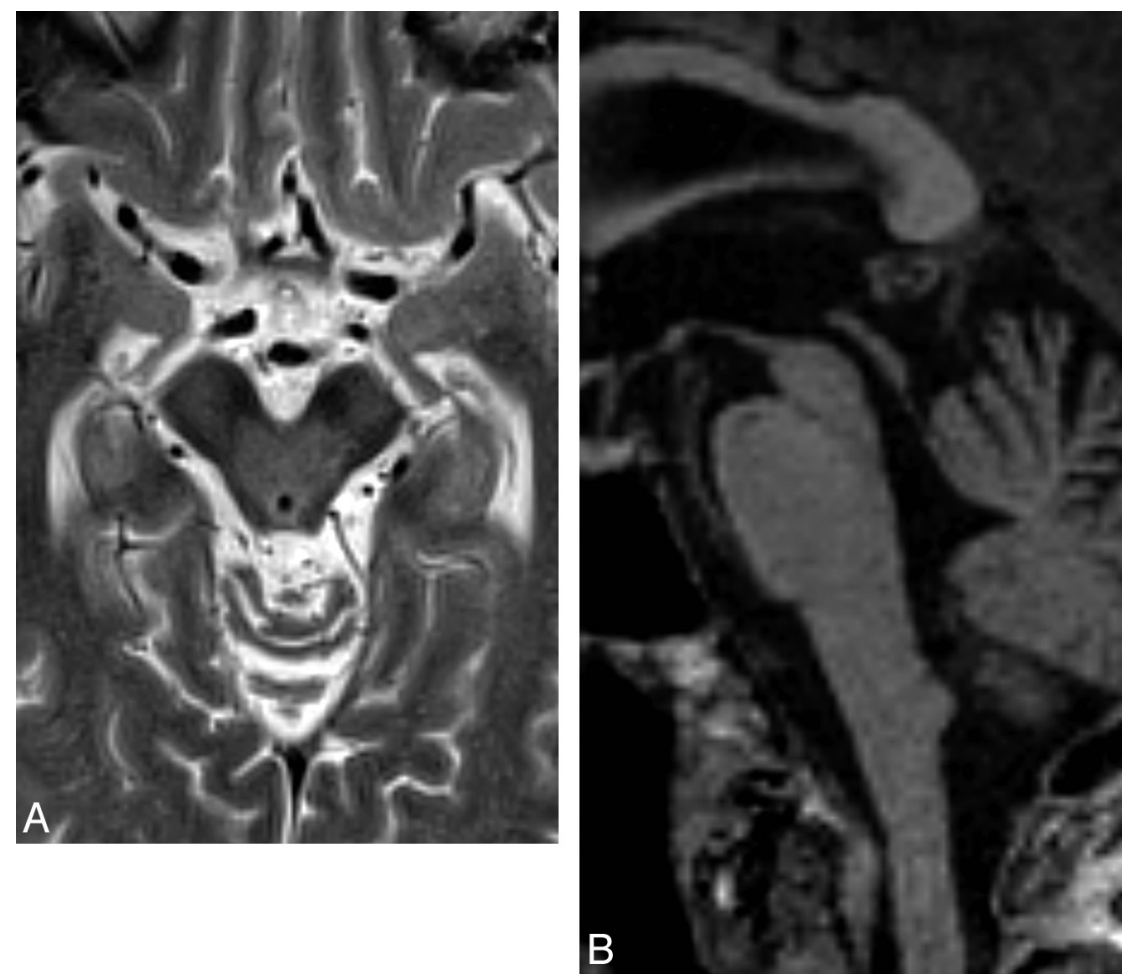

FIG 4. Axial T2-weighted $M R$ imaging sequence in a patient with PSP $(A)$ demonstrating increased midbrain signal intensity (which is only $60 \%$ sensitive and $70 \%$ specific compared with MSA) and an atrophic midbrain with an anteroposterior diameter of $<17 \mathrm{~mm}$ (which is $23 \%$ sensitive and $96 \%$ specific compared with MSA). ${ }^{5}$ The signs are nonspecific in the wider population-for example, midbrain signal intensity can be seen in Wilson disease and a severely atrophic midbrain can be seen in CBD. The concave posterolateral borders of the atrophic midbrain give it a "morning glory" flower appearance. The corresponding sagittal T1-weighted sequence $(B)$ shows tectal atrophy with a "hummingbird" appearance.

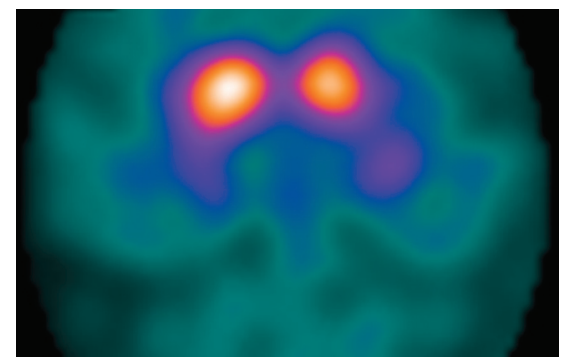

FIG 5. Axial ${ }^{123}$ I-FP-CIT DaT-SPECT image in a patient with PSP, depicting a bilateral reduction in putaminal uptake. Note that the imaging features are similar to those of idiopathic Parkinson disease and MSA.

phometry, respectively, differentiate those with PSP from controls. ${ }^{9,28}$ Additionally, white matter atrophy in the subthalamic region can distinguish PSP from controls and idiopathic Parkinson disease when using voxel-based morphometry. ${ }^{29}$

With similarities to MSA, diffusion imaging and PET may have roles in diagnosing PSP. ADC is increased in the decussation of the superior cerebellar peduncles compared with idiopathic Parkinson disease, MSA, and control groups, ${ }^{30}$ and elevated putaminal ADC might discriminate PSP and idiopathic Parkinson disease with a positive predictive value of $100 \%{ }^{31}$ With PET, ${ }^{11} \mathrm{C}$-raclopride demonstrates reduced striatal dopamine receptor binding, ${ }^{32}$ while ${ }^{18} \mathrm{~F}-\mathrm{FDG}$ is reduced in the putamen, thalamus, frontal cortex, and midbrain. ${ }^{33}$

\section{DaT-SPECT in Progressive Supranuclear Palsy}

In PSP, some DaT-SPECT studies show a predominant loss of DaT density in the putamen, comparable with idiopathic Parkinson disease, ${ }^{17}$ whereas others demonstrate more uniform DaT loss in both the caudate nucleus and putamen. ${ }^{34}$ Overall, on the basis of DaT-SPECT results alone, it is not possible to reliably distinguish idiopathic Parkinson disease, MSA, and PSP (Fig 5) ${ }^{17,18}$ As with MSA, there appears to be $\mathrm{D}_{2}$ receptor striatal loss in PSP, and the diagnostic accuracy of SPECT to differentiate MSA from PSP is low. ${ }^{24}$

In some cases of MSA and PSP, the postsynaptic tracer has normal uptake. As such, a meta-analysis on the diagnostic accuracy of SPECT in parkinsonian syndromes found that postsynaptic and presynaptic tracers could not reliably distinguish idiopathic Parkinson disease and MSA or PSP. ${ }^{35}$ Nonetheless, while negative postsynaptic SPECT scan findings do not exclude MSA or PSP, the positive predictive value of abnormal postsynaptic SPECT findings for the diagnosis of these conditions is very high; therefore, reduced postsynaptic radiotracer binding effectively excludes a diagnosis of idiopathic Parkinson disease.

Some studies combining DaT-SPECT with IBZM or IBF have shown that idiopathic Parkinson disease can be differentiated from MSA or PSP, ${ }^{36,37}$ whereas others have demonstrated otherwise. ${ }^{38,39}$ More promising is a recent prospective study, which, by using a combination of DaT-SPECT, ${ }^{123} \mathrm{I}-$ IBZM SPECT, and meta- ${ }^{123}$ I-iodobenzylguanidine scintigraphy (which probes myocardial adrenergic denervation seen in idiopathic Parkinson disease), reports a positive predictive value of $89 \%$, and a negative predictive value of $97 \%$ (Fig 6). ${ }^{40}$

\section{Corticobasal Degeneration}

$\mathrm{CBD}$ is a rare movement disorder presenting with rigidity, hypokinesia, and predominantly asymmetric cortical dysfunction. It is characterized by deposition of tau proteins in the form of neuronal inclusion bodies. On conventional MR imaging, asymmetric frontoparietal atrophy might distinguish patients with CBD from those with idiopathic Parkinson disease or MSA (Fig 7). ${ }^{26}$ To differentiate CBD from PSP, another tauopathy with frontal atrophy, measuring cortical atrophy with voxel-based morphometry or volumetry appears promising, ${ }^{41,42}$ as does probing motor thalamic involvement with ADC. ${ }^{43}$ With ${ }^{18} \mathrm{~F}$-FDG there is asymmetric hypometabolism in the parietal lobe, while striatal dopamine-receptor binding is relatively preserved. ${ }^{44}$ Transcranial sonography shows substantia nigra hyperechogenicity but does not allow discrimination between idiopathic Parkinson disease and CBD or dementia with Lewy bodies. ${ }^{14}$

AJNR Am J Neuroradiol 36:236-44 Feb 2015 www.ajnr.org 


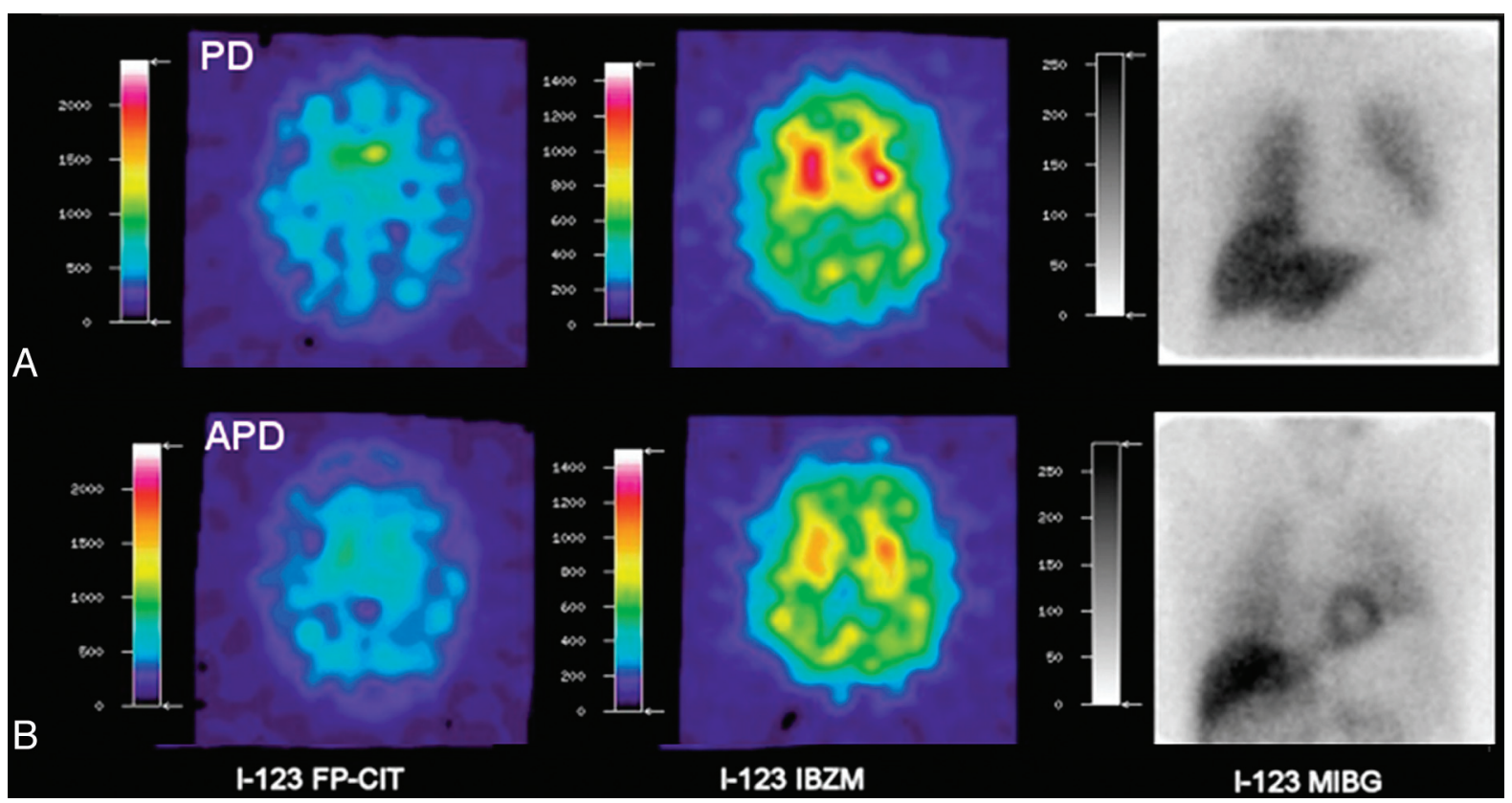

FIG 6. Axial ${ }^{123}$ I-FP-CIT DaT-SPECT, ${ }^{123}$ I-IBZM, and meta- ${ }^{123}$ I-iodobenzylguanidine SPECT studies in idiopathic Parkinson disease (A) and atypical Parkinson disease (MSA or PSP) (B). Uptake patterns with all 3 radiopharmaceuticals are typical for idiopathic Parkinson disease with asymmetric striatal reduction using ${ }^{123} \mathrm{I}$-FP-CIT, normal dopamine $\mathrm{D}_{2}$ receptor binding with ${ }^{123} \mathrm{I}$-IBZM, and reduction in myocardial uptake due to adrenergic denervation with meta- ${ }^{123}$ I-iodobenzylguanidine $(A)$. $B$, Images demonstrate findings of MSA or PSP: reduced striatal uptake with ${ }^{123}$ I-FP-CIT and ${ }^{123}$ I-IBZM but normal myocardial uptake with meta- ${ }^{123}$ I-iodobenzylguanidine. Image courtesy of Dr M. Südmeyer. This was originally published in: Südmeyer M, Antke C, Zizek T, et al. Diagnostic accuracy of combined FP-CIT, IBZM, and MIBG scintigraphy in the differential diagnosis of degenerative parkinsonism: a multidimensional statistical approach. J Nucl Med 2011;52:733-40. ๑ by the Society of Nuclear Medicine and Molecular Imaging, Inc.
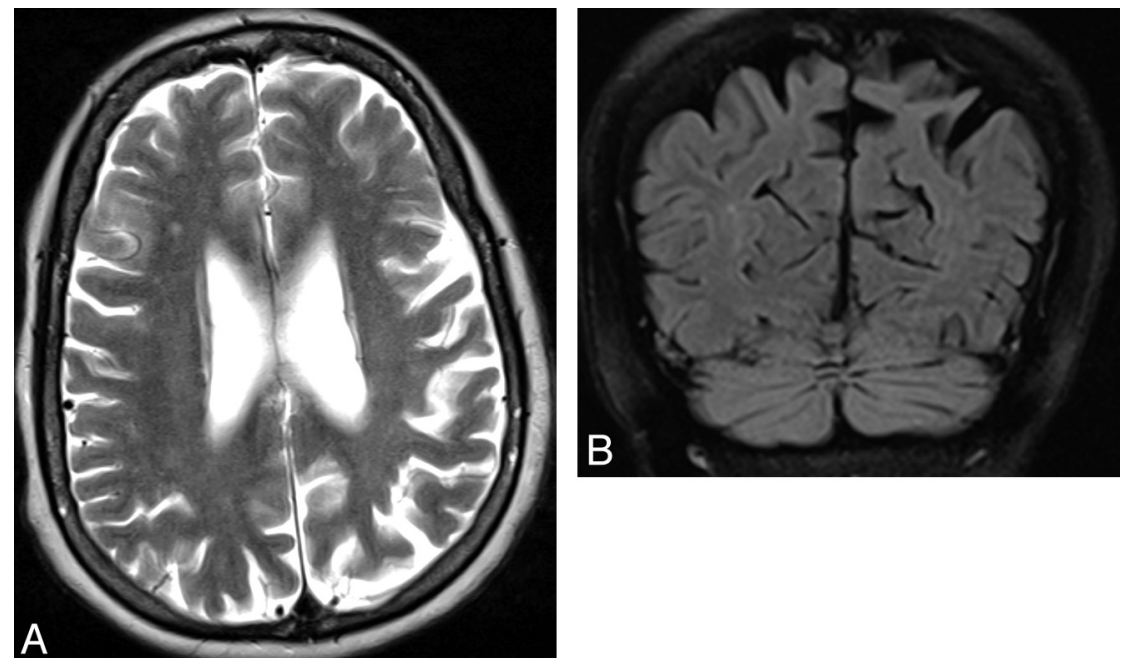

FIG 7. Axial T2-weighted MR imaging sequence in a patient with $C B D(A)$ demonstrating asymmetric frontoparietal atrophy. The corresponding coronal FLAIR sequence $(B)$ shows the asymmetric superior parietal lobules.

\section{DaT-SPECT in Corticobasal Degeneration}

In CBD, the dopaminergic neurons of $10 \%$ of cases appear to have preserved DaT density, whereas the remainder of cases that are affected tend to show a more uniform DaT reduction throughout the striatum and greater hemispheric asymmetry compared with idiopathic Parkinson disease (Fig 8). ${ }^{45}$ However, DaT-SPECT does not allow CBD to be distinguished from idiopathic Parkinson disease, PSP, MSA, and dementia with Lewy bodies at the individual level. ${ }^{39}$ SPECT imaging of the $\mathrm{D}_{2}$ receptor is of limited value, with studies showing either normal or slightly reduced $D_{2}$ receptor binding. ${ }^{39,46}$

\section{Dementia with Lewy Bodies}

Dementia with Lewy bodies is a form of dementia that is thought to be related to idiopathic Parkinson disease. In contrast to idiopathic Parkinson disease, Lewy bodies are found not only in the deep gray matter but diffusely throughout the brain including the cortex. It is the second most common cause of dementia after Alzheimer disease, and distinguishing the 2 diseases is often a diagnostic challenge because the clinical features can overlap. Alzheimer disease accounts for 31\% of those with dementia, presenting with short-term memory decline, language dysfunction, neuropsychiatric features, and, in $5 \%$ of cases, parkinsonism. ${ }^{47-49}$ Dementia with Lewy bodies accounts for approximately $11 \%$ of patients with dementia and can present with falls or depression, which are also seen in Alzheimer disease; fluctuating dementia; and the more discriminatory presentations of visual hallucinations, multiple features, and, in $41 \%$ of cases, parkinsonism. Of note, the clinical features of parkinsonism in both forms of dementia are less severe than those seen in idiopathic Parkinson disease, presenting commonly with rigidity and bradykinesia and infrequently with resting tremor. ${ }^{50}$

It is difficult to differentiate dementia with Lewy bodies from other dementias, though by using MR imaging to differentiate dementia with Lewy bodies from Alzheimer disease and vascular 
dementia, the absence of medial temporal lobe atrophy is highly suggestive of dementia with Lewy bodies. ${ }^{51}$ With voxel-based morphometry, compared with Alzheimer disease, atrophy in the midbrain, hypothalamus, and substantia innominata is marked and the medial temporal lobe is relatively preserved. ${ }^{52}$ Nonetheless, medial temporal lobe atrophy often occurs in dementia with Lewy bodies, with volumetry showing the entorhinal cortex to be

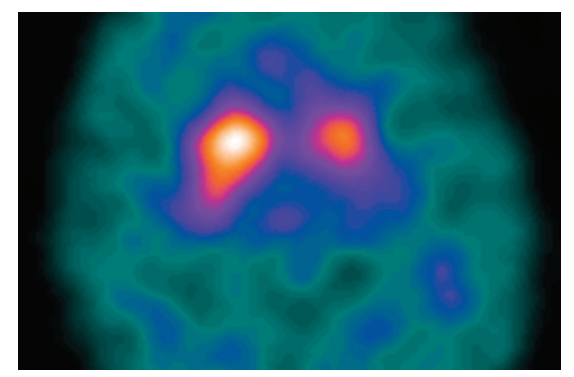

FIG 8. Axial ${ }^{123}$ I-FP-CIT DaT SPECT image in a patient with corticobasal degeneration. There is uniform striatal reduction in tracer activity with marked hemispheric asymmetry on the left. However, in many cases, imaging appearances can be indistinguishable from those in idiopathic Parkinson disease, MSA, or PSP. Image courtesy of Dr Roberto Ceravolo, Department of Neurosciences, University of Pisa, Italy. ${ }^{45}$
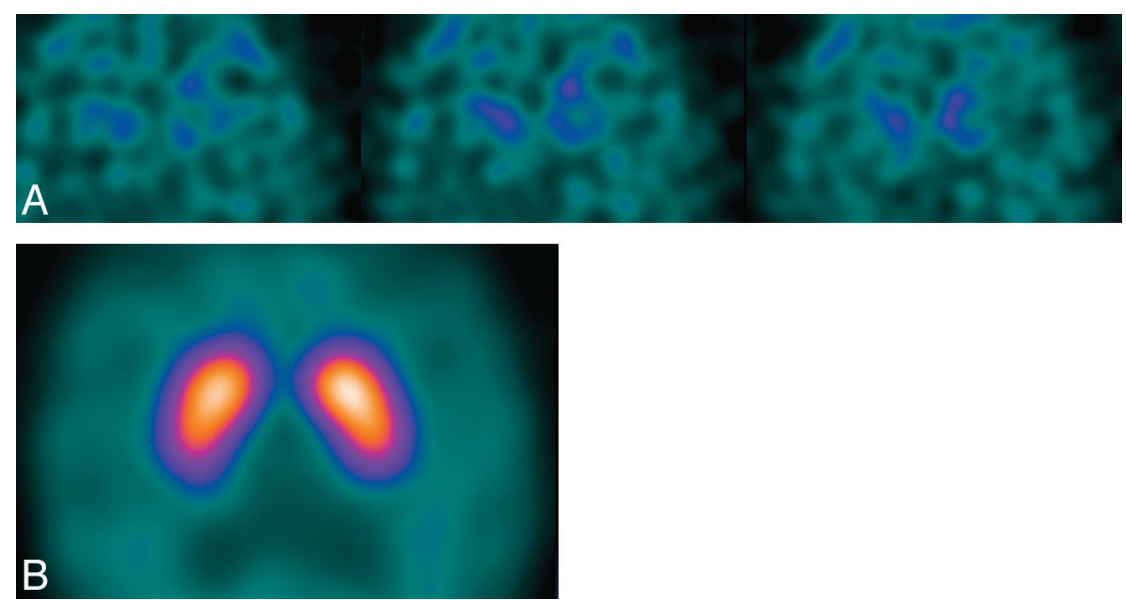

FIG 9. Axial ${ }^{123}$ I-FP-CIT DaT-SPECT images in a patient with dementia with Lewy bodies $(A)$ and a patient with Alzheimer disease (B). Note that the DaT-SPECT study helped to distinguish the 2 causes of dementia with the patient with dementia with Lewy bodies exhibiting a Catafau and Tolosa ${ }^{2}$ type 3 pattern of abnormal striatal uptake; in contrast, the patient with Alzheimer disease demonstrates normal striatal uptake. Images from both patients have low gain as demonstrated by low background activity.
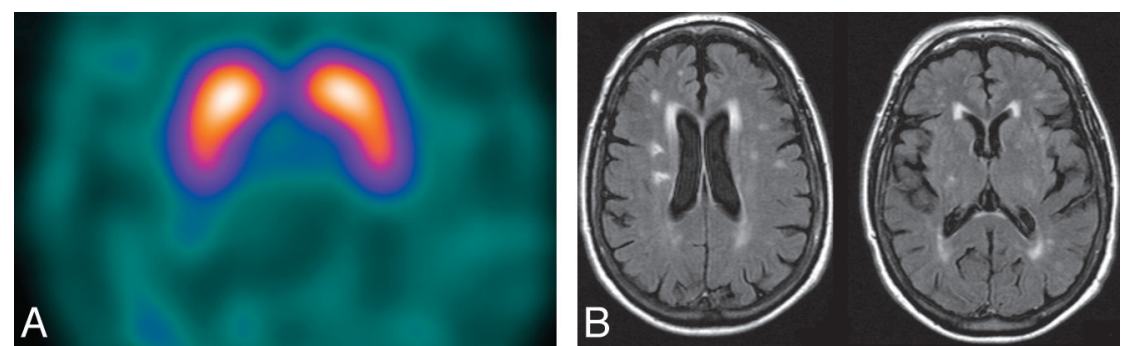

FIG 10. Axial ${ }^{123}$ I-FP-CIT DaT-SPECT $(A)$ and axial MR imaging FLAIR sequence $(B)$ of the brain in the same patient with vascular parkinsonism. Note that the DAT-SPECT study has normal findings with symmetric, intense uptake in the caudate nuclei and putamina. This effectively excludes a nigrostriatal degenerative cause of parkinsonism. Consistent with ischemic disease, the MR imaging demonstrates diffuse T2-weighted hyperintensities in the striatum and in the periventricular and subcortical white matter. smaller than that in control and idiopathic Parkinson disease groups. ${ }^{53}$ In the parietal lobe of those with dementia with Lewy bodies, DTI appears to show reduced fractional anisotropy comwith control or Alzheimer disease groups. ${ }^{54}$ While in the hypometabolism are demonstrated by reduced hexamethylpro-

\section{DaT-SPECT in Dementia with Lewy Bodies}

ortem studies have shown normal levels of putaminal dodementia with Lewy bodies. SPECT studies that show an asymmetric reduction in DAT binding in dementia with Lewy bodies compared with a normal appearance in Alzheimer disease, allowing differentiation with $90 \%$ positive predictive value (Fig 9). ${ }^{39,58}$ It is particularly important to some neuroleptic drugs block the postsynaptic $\mathrm{D}_{2}$ receptor, thereby exacerbating the shortage of dopamine within the synaptic cleft. More than half of such patients will have severe adverse reactions, some of which are fatal. ${ }^{49}$

DaT-SPECT does not allow idiopathic Parkinson disease with dementia (an arbitrary definition of dementia developing at least 1 year after the diagnosis of idiopathic Parkinson disease) and dementia with Lewy bodies to be distinguished. ${ }^{58}$

\section{Other DaT SPECT Imaging Features}

Idiopathic Parkinson Disease versus Vascular Parkinsonism. Because vascular lesions are often incidental findings in patients with idiopathic Parkinson disease, it is often difficult to diagnose parkinsonism secondary to vascular lesions (vascular parkinsonism). Criteria for the diagnosis of vascular parkinsonism have been described by Zijlmans et $\mathrm{al}^{59}$ following a detailed clinical and neuropathologic analysis of the condition. The authors proposed that the diagnosis of vascular parkinsonism could be made if the following clinicoradiologic features were present: 1) bradykinesia with at least 1 of the following: resting tremor, rigidity, or postural instability; 2) cerebrovascular disease demonstrated either on CT/MR imaging or by the presence of focal clinical signs of stroke; and 3) a relationship between the 2 disorders: specifically, an acute or delayed progressive onset with infarcts in or near areas that can increase the basal ganglia motor output (globus 
pallidus or substantia nigra pars compacta) or decrease the thalamocortical drive directly (thalamus or large frontal lobe infarct), or an insidious onset of parkinsonism with extensive subcortical white matter lesions, bilateral symptoms at onset, and the presence of an early shuffling gait or cognitive dysfunction.

These criteria, even when applied to the retrospective sample from which they were derived, are not $100 \%$ sensitive. DaTSPECT has, therefore, been used to help establish the diagnosis of vascular parkinsonism because the nigrostriatal dopaminergic pathway is typically unaffected, ${ }^{59}$ and consequently, the DaT binding will not be reduced as in idiopathic Parkinson disease. ${ }^{39}$ The caveat is that occasionally a massive vascular infarct within the striatum can cause moderate nigrostriatal degeneration ${ }^{60}$; therefore, though infrequent, marked asymmetry can be evident on DaT-SPECT. Nonetheless, in the absence of postsynaptic causes of parkinsonism (eg, drug-induced) and the presence of vascular lesions on CT/MR imaging (eg, a "punched out" lesion characteristic of an infarct or the diffuse white matter disease of ischemia), in an appropriate clinical context, normal DaT-SPECT

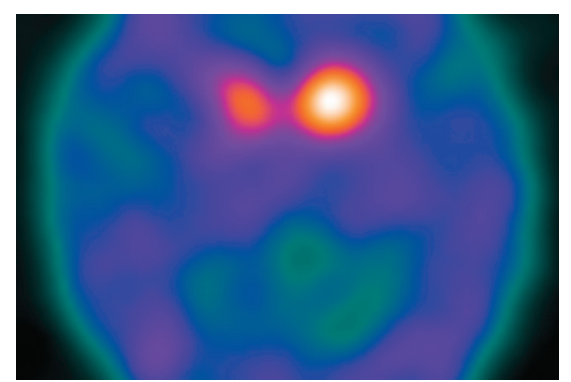

FIG 11. Axial ${ }^{123}$ I-FP-CIT DaT-SPECT image from a patient with schizophrenia on neuroleptic medication. The patient presented with extrapyramidal signs. If the patient's clinical signs were purely due to the side effects of neuroleptic medication, then the DaT-SPECT study findings would be normal. However, in this case, there is absent putaminal uptake bilaterally and asymmetric caudate nuclei uptake (Catafau and Tolosa ${ }^{2}$ type 2), indicative of coexistent idiopathic Parkinson disease, MSA, or PSP.
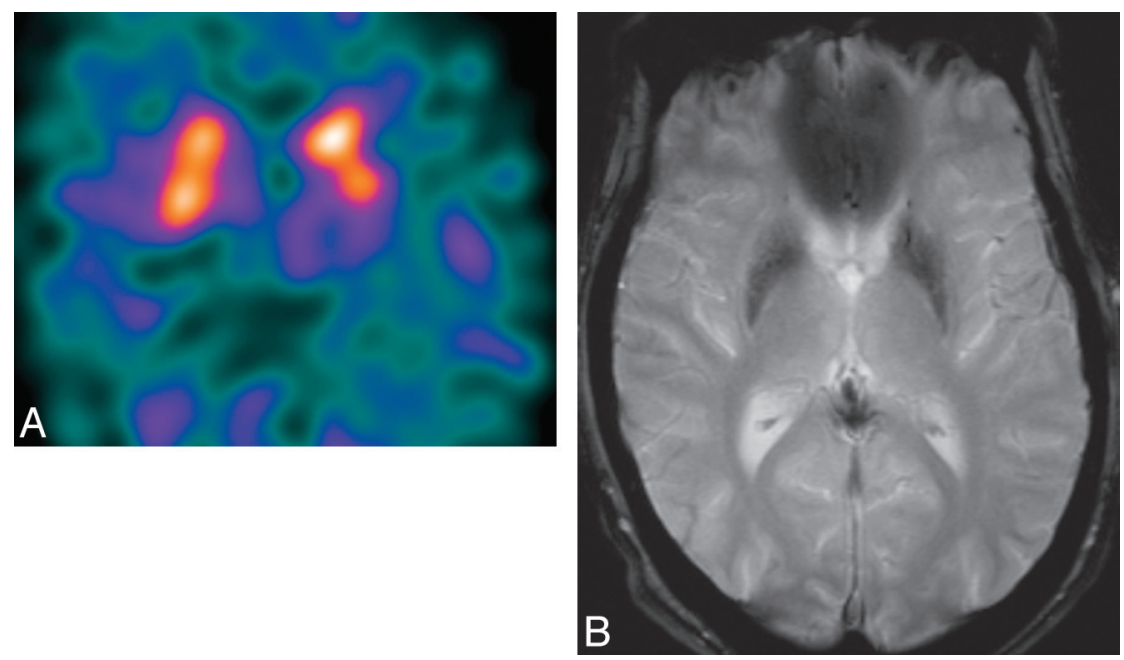

FIG 12. Axial ${ }^{123}$ I-FP-CIT DaT-SPECT image $(A)$ and axial T2*-weighted MR imaging sequence $(B)$ in the same patient with neuroacanthocytosis. The patient presented with parkinsonian features. Note the asymmetric reduction of ${ }^{123}$ I-FP-CIT uptake in the left putamen. MR imaging depicts marked hypointensity in the caudate nuclei and putamina, indicative of iron deposition, which is a feature of neuroacanthocytosis. findings are diagnostic of vascular parkinsonism (Fig 10). ${ }^{1}$ Overall, a meta-analysis of the diagnostic differentiation of idiopathic Parkinson disease and vascular parkinsonism by using DaTSPECT demonstrated a sensitivity of $80 \%-100 \%$ and a specificity of $73 \%-100 \% .^{38}$

Idiopathic Parkinson Disease versus Drug-Induced Parkinsonism. Drugs that have a competitive antagonistic effect on the postsynaptic $\mathrm{D}_{2}$ receptors, such as neuroleptics (eg, olanzapine) and centrally acting antiemetics (eg, metoclopramide), can cause parkinsonism, which is usually reversible. Clinically, it can be very difficult to distinguish drug-induced parkinsonism from idiopathic Parkinson disease. ${ }^{1}$ However, in contrast to idiopathic Parkinson disease, DaT-SPECT imaging has either normal findings or depicts increased striatal DAT activity, the latter suggesting a compensatory up-regulation of $\mathrm{DaT}$ in response to $D_{2}$ receptor blockade. ${ }^{38,61}$ Of clinical importance is the masking of early idiopathic Parkinson disease in patients with schizophrenia on neuroleptic medication. In these cases, the DaT-SPECT study findings will be abnormal, in keeping with coexistent idiopathic Parkinson disease (or another neurodegenerative parkinsonian syndrome) (Fig 11).

Idiopathic Parkinson Disease and Essential Tremor. Typically, the unilateral resting tremor of idiopathic Parkinson disease can be distinguished from the symmetric essential tremor on clinical grounds, especially when there are other signs of parkinsonism. However, clinical confusion may occur when the 2 diagnoses coexist or when there are equivocal parkinsonian features. In these cases, DaT-SPECT discriminates essential tremor and idiopathic Parkinson disease because patients with essential tremor consistently have, effectively, normal striatal DaT binding. ${ }^{18,38,39,62}$ Indeed, in a meta-analysis of the diagnostic differentiation of essential tremor and idiopathic Parkinson disease using DaTSPECT, the study with the lowest sensitivity and specificity reported percentages of $80 \%$ and $95 \%$, respectively. ${ }^{35}$ Recently, DaT-SPECT has challenged the view that essential tremor has absolutely no pathophysiologic effect on dopaminergic transmission because a slight reduction in caudate DaT binding has been noted. ${ }^{62}$

\section{Rare Causes of Parkinsonism}

Nigrostriatal degeneration may also be seen in other, rarer, causes of parkinsonism. Wilson disease demonstrates striatal DaT-SPECT reduction comparable with that in idiopathic Parkinson disease. ${ }^{63}$ Similarly, reductions in DaT binding are seen in sporadic amyotrophic lateral sclerosis. ${ }^{64}$ Neuroacanthocytosis encompasses a heterogeneous group of disorders characterized by the association of neurologic abnormalities with red cell acanthocytosis. They are categorized into 2 groups based on the presence or absence of movement disorders. In patients with neuroacanthocytosis presenting with movement disorders, there is underlying degeneration of the striatum. ${ }^{65}$ There- 
fore, it can be inferred that DaT-SPECT imaging is likely to be abnormal in these cases (Fig 12).

In conclusion, dopamine-transporter imaging, in particular DaT-SPECT, reliably distinguishes neurodegenerative causes of parkinsonism (idiopathic Parkinson disease, MSA, PSP, and CBD) from parkinsonism without nigrostriatal DaT loss (such as essential tremor, vascular parkinsonism, and drug-induced parkinsonism). DaT-SPECT alone cannot differentiate the disorders within the neurodegenerative group, whereas initial studies using a combination of radiotracers or other advanced imaging techniques appear promising. DaT-SPECT also helps to differentiate dementia secondary to Lewy body deposition and Alzheimer disease, thereby avoiding a potential treatment-induced parkinsonian crisis. It is hoped that this technique will be the forerunner of a range of routinely used, process-specific ligands that can identify early degenerative disease and subsequently guide disease-modifying interventions.

\section{ACKNOWLEDGMENTS}

The authors thank Dr Jonathan Hart for help in manuscript preparation.

Disclosures: Ann-Marie Quigley—RELATED: Other: Travel, hotel and conference fees were paid by Novartis to attend the European Neuroendocrine Tumor Society 2013 meeting; travel and hotel paid by Nordion to attend the TheraSphere User Group in 2013; No fees were paid to me or my institution. Adam D. WaldmanUNRELATED: Consultancy: Bayer Healthcare, Payment for Lectures (including service on Speakers Bureaus): Bayer Healthcare, Siemens Healthcare, Comments: fees for lectures on education courses, Travel/Accommodations/Meeting Expenses Unrelated to Activities Listed: Bayer Healthcare, Comments: expenses for attendance at meetings for discussion groups and consultancy. John Buscombe-UNRELATED: Consultancy: Navidea, Comments: for work on Medicines and Healthcare Products Regulatory Agency submission, Travel/Accommodations/Meeting Expenses Unrelated to Activities Listed: BTG, Comments: to learn about TheraSpheres, Germany, February 2014. Anthony H. Schapira—RELATED: receives Medical Research Council and Wellcome Trust funding.

\section{REFERENCES}

1. Scherfler C, Schwarz J, Antonini A, et al. Role of DAT-SPECT in the diagnostic work-up of parkinsonism. Mov Disord 2007;22:1229-38

2. Catafau AM, Tolosa E. Impact of dopamine transporter SPECT using ${ }^{123}$ I-Ioflupane on diagnosis and management of patients with clinically uncertain parkinsonian syndromes. Mov Disord 2004;19:1175-82

3. Quinn N. Multiple system atrophy: the nature of the beast. J Neurol Neurosurg Psychiatry 1989(suppl):78-89

4. Papp MI, Lantos PL. The distribution of oligodendroglial inclusions in multiple system atrophy and its relevance to clinical symptomatology. Brain 1994;117:235-43

5. Schrag A, Good CD, Miszkiel K, et al. Differentiation of atypical parkinsonian syndromes with routine MRI. Neurology 2000;54: 697-702

6. Nicoletti G, Fera F, Condino F, et al. MR imaging of middle cerebellar peduncle width: differentiation of multiple system atrophy from Parkinson disease. Radiology 2006;239:825-30

7. Ghaemi M, Hilker R, Rudolf J, et al. Differentiating multiple system atrophy from Parkinson's disease: contribution of striatal and midbrain MRI volumetry and multi-tracer PET. J Neurol Neurosurg Psychiatry 2002;73:517-23

8. Brenneis C, Seppi K, Schocke MF, et al. Voxel-based morphometry detects cortical atrophy in the Parkinson variant of multiple system atrophy. Mov Disord 2003;18:1132-38

9. Schulz JB, Skalej M, Wedekind D, et al. Magnetic resonance imagingbased volumetry differentiates idiopathic Parkinson's syndrome from multiple system atrophy and progressive supranuclear palsy. Ann Neurol 1999;45:65-74

10. Schocke MF, Seppi K, Esterhammer R, et al. Diffusion-weighted MRI differentiates the Parkinson variant of multiple system atrophy from PD. Neurology 2002;58:575-80

11. Nicoletti G, Lodi R, Condino F, et al. Apparent diffusion coefficient measurements of the middle cerebellar peduncle differentiate the Parkinson variant of MSA from Parkinson's disease and progressive supranuclear palsy. Brain 2006;129:2679-87

12. Ito $M$, Watanabe $H$, Kawai $Y$, et al. Usefulness of combined fractional anisotropy and apparent diffusion coefficient values for detection of involvement in multiple system atrophy. J Neurol Neurosurg Psychiatry 2007;78:722-28

13. Watanabe $H$, Fukatsu $H$, Katsuno M, et al. Multiple regional 1H-MR spectroscopy in multiple system atrophy: NAA/Cr reduction in pontine base as a valuable diagnostic marker. J Neurol Neurosurg Psychiatry 2004;75:103-09

14. Berg D, Godau J, Walter U. Transcranial sonography in movement disorders. Lancet Neurol 2008;7:1044-55

15. Brooks DJ, Salmon EP, Mathias CJ, et al. The relationship between locomotor disability, autonomic dysfunction, and the integrity of the striatal dopaminergic system in patients with multiple system atrophy, pure autonomic failure, and Parkinson's disease, studied with PET. Brain 1990;113:1539-52

16. Antonini A, Leenders KL, Vontobel P, et al. Complementary PET studies of striatal neuronal function in the differential diagnosis between multiple system atrophy and Parkinson's disease. Brain 1997;120:2187-95

17. Brücke T, Asenbaum S, Pirker W, et al. Measurement of the dopaminergic degeneration in Parkinson's disease with [123I] beta-CIT and SPECT: correlation with clinical findings and comparison with multiple system atrophy and progressive supranuclear palsy. $\mathrm{JNeu}$ ral Transm Suppl 1997;50:9-24

18. Benamer TS, Patterson J, Grosset DG, et al. Accurate differentiation of parkinsonism and essential tremor using visual assessment of $\left[{ }^{123} \mathrm{I}\right]$-FP-CIT SPECT imaging: the $\left[{ }^{123} \mathrm{I}\right]$-FP-CIT study group. Mov Disord 2000;15:503-10

19. Nocker M, Seppi K, Donnemiller E, et al. Progression of dopamine transporter decline in patients with the Parkinson variant of multiple system atrophy: a voxel-based analysis of [123I]beta-CIT SPECT. Eur J Nucl Med Mol Imaging 2012;39:1012-20

20. Seeman P, Niznik H. Dopamine receptors and transporters in Parkinson's disease and schizophrenia. FASEB J 1990;4:2737-44

21. Costa DC, Verhoeff NP, Cullum ID, et al. In vivo characterisation of 3-iodo-6-methoxybenzamide ${ }^{123} \mathrm{I}$ in humans. Eur $\mathrm{J} \mathrm{Nucl} \mathrm{Med}$ 1990;16:813-16

22. Ichise M, Ballinger JR, Vines D, et al. Simplified quantification and reproducibility studies of dopamine $\mathrm{D}_{2}$-receptor binding with iodine-123-IBF SPECT in healthy subjects. J Nucl Med 1997;38:31-37

23. Churchyard A, Donnan GA, Hughes A, et al. Dopa resistance in multiple-system atrophy: loss of postsynaptic $\mathrm{D}_{2}$ receptors. Ann Neurol 1993;34:219-26

24. van Royen E, Verhoeff NPLG, Speelman JD, et al. Multiple system atrophy and progressive supranuclear palsy: diminished striatal $\mathrm{D}_{2}$-receptor activity demonstrated by ${ }^{123} \mathrm{I}$-IBZM single photon emission computed tomography. Arch Neurol 1993;50:513-16

25. Schwarz J, Tatsch K, Gasser T, et al. 123I-IBZM binding compared with long-term clinical follow up in patients with de novo parkinsonism. Mov Disord 1998;13:16-19

26. Yekhlef F, Ballan G, Macia F, et al. Routine MRI for the differential diagnosis of Parkinson's disease, MSA, PSP, and CBD. J Neural Transm 2003;110:151-69

27. Quattrone A, Nicoletti G, Messina D, et al. MR imaging index for differentiation of progressive supranuclear palsy from Parkinson disease and the Parkinson variant of multiple system atrophy. $R a$ diology 2008;246:214-21

28. Brenneis C, Seppi K, Schocke MF, et al. Voxel-based morphometry 
reveals a distinct pattern of frontal atrophy in progressive supranuclear palsy. J Neurol Neurosurg Psychiatry 2004;75:246-49

29. Price S, Paviour D, Scahill R, et al. Voxel-based morphometry detects patterns of atrophy that help differentiate progressive supranuclear palsy and Parkinson's disease. Neuroimage 2004;23: 663-69

30. Blain CR, Barker GJ, Jarosz JM, et al. Measuring brain stem and cerebellar damage in parkinsonian syndromes using diffusion tensor MRI. Neurology 2006;67:2199-205

31. Seppi K, Schocke MF, Esterhammer R, et al. Diffusion-weighted imaging discriminates progressive supranuclear palsy from PD, but not from the Parkinson variant of multiple system atrophy. Neurology 2003;60:922-27

32. Brooks DJ, Ibanez V, Sawle GV, et al. Striatal $\mathrm{D}^{2}$ receptors in patients with Parkinson's disease, striatonigral degeneration, and progressive supranuclear palsy, measured with ${ }^{11} \mathrm{C}$-raclopride and positron emission tomography. Ann Neurol 1992;31:184-92

33. Garraux G, Salmon E, Degueldre C, et al. Comparison of impaired subcortico-frontal metabolic networks in normal aging, subcortico-frontal dementia, and cortical frontal dementia. Neuroimage 1999;10:149-62

34. Antonini A, Benti R, De NR, et al. ${ }^{123}$ I-Ioflupane SPECT binding to striatal dopamine transporter (DAT) uptake in patients with Parkinson's disease, multiple system atrophy and progressive supranuclear palsy. Neurol Sci 2003;24:149-50

35. Vlaar AM, van Kroonenburgh MJ, Kessels AG, et al. Meta-analysis of the literature on the diagnostic accuracy of SPECT in parkinsonian syndromes. BMC Neurol 2007;1:27

36. Koch W, Hamann C, Radau PE, et al. Does combined imaging of the pre-and postsynaptic dopaminergic system increase the diagnostic accuracy in the differential diagnosis of parkinsonism? Eur J Nucl Med Mol Imaging 2007;34:1265-73

37. Kim YJ, Ichise M, Ballainger JR, et al. Combination of dopamine transporter and $D_{2}$ receptor SPECT in the diagnostic evaluation of PD, MSA and PSP. Mov Disord 2002;2:303-12

38. Vlaar AM, de Nijs T, Kessels AG, et al. Diagnostic value of ${ }^{123}$ I-Ioflupane and ${ }^{123}$ I-iodobenzamide SPECT scans in 248 patients with Parkinsonian syndromes. Eur Neurol 2008;59:258-66

39. Plotkin M, Amthauer H, Klaffke S, et al. Combined $\left[{ }^{123} \mathrm{I}\right]-$ FPCIT and $\left[{ }^{123} \mathrm{I}\right]$-IBZM SPECT for the diagnosis of parkinsonian syndromes: study on 72 patients. J Neural Transm 2005;112:677-92

40. Südmeyer M, Antke C, Zizek T, et al. Diagnostic accuracy of combined FP-CIT, IBZM, and MIBG scintigraphy in the differential diagnosis of degenerative parkinsonism: a multidimensional statistical approach. J Nucl Med 2011;52:733-40

41. Gröschel K, Hauser TK, Luft A, et al. Magnetic resonance imagingbased volumetry differentiates progressive supranuclear palsy from corticobasal degeneration. Neuroimage 2004;21:714-24

42. Boxer AL, Geschwind MD, Belfor N, et al. Patterns of brain atrophy that differentiate corticobasal degeneration syndrome from progressive supranuclear palsy. Arch Neurol 2006;63:81-86

43. Erbetta A, Mandelli ML, Savoiardo M, et al. Diffusion tensor imaging shows different topographic involvement of the thalamus in progressive supranuclear palsy and corticobasal degeneration. AJNR Am J Neuroradiol 2009;30:1482-87

44. Klaffke S, Kuhn AA, Plotkin M, et al. Dopamine transporters, D2 receptors, and glucose metabolism in corticobasal degeneration. Mov Disord 2006;21:1724-27

45. Cilia R, Rossi C, Frosini D, et al. Dopamine transporter SPECT imaging in corticobasal syndrome. PLoS One 2011;6:e18301

46. Pirker S, Perju-Dumbrava L, Kovacs GG, et al. Dopamine D2 recep- tor SPECT in corticobasal syndrome and autopsy-confirmed corticobasal degeneration. Parkinsonism Relat Disord 2013;19:222-26

47. Stevens $T$, Livingston $G$, Kitchen $G$, et al. Islington study of dementia subtypes in the community. Br J Psychiatry 2002;180:270-76

48. Ala TA, Yang KH, Sung JH, et al. Hallucinations and signs of parkinsonism help distinguish patients with dementia and cortical Lewy bodies from patients with Alzheimer's disease at presentation: a clinicopathological study. J Neurol Neurosurg Psychiatry 1997; 62:16-21

49. McKeith I, Fairbairn A, Perry R, et al. Neuroleptic sensitivity in patients with senile dementia of Lewy body type. BMJ 1992;305: 673-78

50. Ditter SM, Mirra SS. Neuropathologic and clinical features of Parkinson's disease in Alzheimer's disease patients. Neurology 1987;37:754-60

51. Barber R, Gholkar A, Scheltens P, et al. Medial temporal lobe atrophy on MRI in dementia with Lewy bodies. Neurology 1999;52:1153-58

52. Whitwell JL, Weigand SD, Shiung MM, et al. Focal atrophy in dementia with Lewy bodies on MRI: a distinct pattern from Alzheimer's disease. Brain 2007;130:708-19

53. Kenny ER, Burton EJ, O’Brien JT. A volumetric magnetic resonance imaging study of entorhinal cortex volume in dementia with Lewy bodies: a comparison with Alzheimer's disease and Parkinson's disease with and without dementia. Dement Geriatr Cogn Disord 2008;26:218-25

54. Firbank MJ, Blamire AM, Krishnan MS, et al. Diffusion tensor imaging in dementia with Lewy bodies and Alzheimer's disease. Psychiatry Res 2007;155:135-45

55. Lobotesis K, Fenwick JD, Phipps A, et al. Occipital hypoperfusion on SPECT in dementia with Lewy bodies but not AD. Neurology 2001;56:643-49

56. Gilman S, Koeppe RA, Little R, et al. Differentiation of Alzheimer's disease from dementia with Lewy bodies utilizing positron emission tomography with [18F]fluorodeoxyglucose and neuropsychological testing. Exp Neurol 2005;191:S95-S103

57. Piggott MA, Marshall EF, Thomas N, et al. Striatal dopaminergic markers in dementia with Lewy bodies, Alzheimer's and Parkinson's diseases: rostrocaudal distribution. Brain 1999;122:1449-68

58. O’Brien JT, Colloby S, Fenwick J, et al. Dopamine transporter loss visualized with FP-CIT SPECT in the differential diagnosis of dementia with Lewy bodies. Arch Neurol 2004;61:919-25

59. Zijlmans JC, Daniel SE, Hughes AJ, et al. Clinicopathological investigation of vascular parkinsonism, including clinical criteria for diagnosis. Mov Disord 2004;19:630 - 40

60. Forno L. Reaction of the substantia nigra to massive basal ganglia infarction. Acta Neuropathol (Berl) 1983;62:96-102

61. Sjøholm H, Bratlid T, Sundsfjord J. [ ${ }^{123}$ I]-beta-CIT SPECT demonstrates increased presynaptic dopamine transporter binding sites in basal ganglia in vivo in schizophrenia. Psychopharmacology (Berl) 2004;173:27-31

62. Isaias IU, Marotta G, Hirano S, et al. Imaging essential tremor. Mov Disord 2010;25:679-86

63. Jeon B, Kim JM, Jeong JM. Dopamine transporter imaging with $\left[{ }^{123} \mathrm{I}\right]$-beta-CIT demonstrates presynaptic nigrostriatal dopaminergic damage in Wilson's disease. J Neurol Neurosurg Psychiatry 1998;65:60-64

64. Borasio GD, Linke R, Schwarz J, et al. Dopaminergic deficit in amyotrophic lateral sclerosis assessed with [I-123] IPT single photon emission computed tomography. J Neurol Neurosurg Psychiatry 1998;65:263-65

65. Ichiba M, Nakamura M, Sano A. Neuroacanthocytosis update. Brain Nerve 2008;60:635-41 